\section{Chaos for oscillating voles}

IT's a tough life, being a vole in northern Fennoscandia. If the harsh climate and the ever. present peril of being eaten by a weasel Mustela nivalis (pictured here) weren't enough, there is now the Lyapunov expo. nent to cope with. For as Ilkka Hanski and colleagues show on page 232 of this issue, the life of the vole is more than

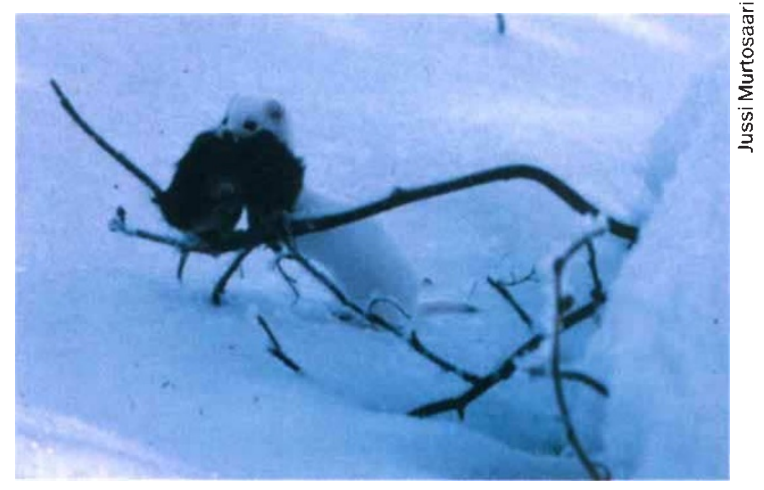
just difficult - it's chaotic. A model of predator-prey interaction, modulated by seasonality, simulates with some accuracy the observed three-to-five year population cycles of northern voles. Put another way, the field data can also be broken down by nonlinear analysis to reveal their underlying chaotic components. Rodent population dynamics, whether predicted or observed, are chaotic, but with a strong periodic component imposed by the density-dependent appetites of their mustelid tormentors. Interestingly, chaos is absent in vole populations further south, where the climate is more clement and the predators more varied. H.G.

Guariglia et al. which yields the opposite result to theirs. But observations by Mesulam $^{4}$ and Anderson ${ }^{5}$ suggest that the reverse result may yet be found.

A traditional test for neglect requires the patient to draw a clockface from memory. The figure on page 193 indicates each of the two results typically found in neglect: the patient either leaves out the numbers on the left, or transposes them to the right side of the clockface. The examples given (from one of our own patients) show omission in the acute phase after stroke, followed by transposition in the chronic phase.

Anderson ${ }^{5}$ has reported a patient with stroke-induced damage of the right parietal lobe and right thalamus who reliably produced clock-drawings of the second (transposed) type.when tested, as is customary, with the eyes open. But when required to perform the same task with the eyes closed, her clockface was drawn normally, with all 12 numbers appropriately placed around the full circumference. Anderson argues (metaphorically) that "right-sided external percepts are more sticky than internal images", but the results of Guariglia et al. (on a different patient) show the right side of images to be the more "sticky".

In the case of Guariglia et al., the site of brain damage was right fronto-temporal. This too is surprising, as most previous studies of the generation of visual imagery have shown that left occipito-temporal regions are maximally involved in this process. These areas were intact in the patient of Guariglia et al. Accordingly, the authors suggest that their patient can generate "the global image of an object", but that this global representation does not suffice to place specific (local) items correctly within the overall space. Other recent results, using conventional cancellation tests for neglect, are compatible can accurately mark the four corners of a sheet of paper that contains small (local) lines pseudorandomly scattered across the full dimensions of the page. Yet immediately afterwards the patient fails to cross out the small lines on the left of the sheet while having no difficulty cancelling those on the right ${ }^{6}$

This interplay between global and local perception provokes a question that no study of imaginal neglect has yet addressed: what shape does the patient of Guariglia et al. imagine the Piazza dei Cinquecento in Rome to be? If global perception and memory are intact, he should draw (or verbally describe) it correctly; if the global image is 'neglected', the piazza should shrink in the lateral dimension. Whatever the result, the evidence is clearly mounting that in neglect, as in the visual agnosias ('seeing without recognizing') ${ }^{7}$, visuospatial perception and visuospatial imagery do not draw upon identical neuronal substrates.

John C. Marshall and Peter W. Halligan are in the Neuropsychology Unit, University Department of Clinical Neurology, Radcliffe Infirmary, Oxford OX2 6HE, UK.

\footnotetext{
1. Guariglia, C., Padovani, A., Pantano, P. \& Pizzamiglio, L. Nature 364, 235-237 (1993).

2. Grusser, O.-J. \&Landis, T. Visual Agnosias and Other Disturbances of Visual Perception and Cognition (Macmilian, London, 1991).

3. Bisiach, E. \& Luzzatti, C. Cortex 14, 129-133 (1978)

4. Mesulam. M.-M. Principles of Behavioral Neurology (Davis, Philadelphia, 1985).

Anderson, B. Neurology 43, 213-216 (1993).

6. Halligan, P. W. \& Marshall, J. C. Cortex 29, 167-174 (1993).

. Gurd, J. M. \& Marshall, J. C. Nature 359, 590-591 (1992)
} with this conjecture. Patients with neglect

\section{DAEDALUS \\ Negative energy}

A HOAX of the 1960 s claimed that some foods, such as hard-boiled eggs, used up more calories in being digested than they provided in food value. So by eating enough of them you could lose weight. Overweight egg-lovers were soon sadly disillusioned; but Daedalus now proposes a food that works this elegant trick in reality.

He points out that digestion is a process of hydrolysis. It breaks down fats, carbohydrates and proteins into their small component molecules simply by reacting them with water. The reaction evolves little energy and is easily reversible, so that the body can later recombine the components into fats, carbohydrates and proteins of its own. DREADCO chemists are devising a fat whose components combine spontaneously. The fatty acid component is sterically designed to enfold the glycerol part; the molecules attract each other and combine rapidly, giving out energy. To hydrolyse them apart, this energy must be supplied again.

Incorporated into cooking oil, chocolate and so on, DREADCO's 'Thinfat' ${ }^{\circledR}$ will transform dieting. The dieter's digestive system will invest the required amount of energy to hydrolyse it to glycerol and free fatty acid. The separated components will then promptly combine again, liberating that energy as heat. The digestive enzymes will set to work to hydrolyse the stuff again, only to have their work promptly undone a second time. The cycle will continue indefinitely. In metabolic terms, Thinfat has a negative calorific value.

A Thinfat user should enjoy his diet. Instead of indigestion, he will have the novel experience of hyperdigestion. His warm and comfortable after-dinner lethargy will persist for hours, as energy is drained from his body and released as heat. It will be a wonderfully lazy way to lose weight.

Even when the digestive system has won its exhausting battle, Thinfat will continue its work. Once inside the body, it will be stored in the normal fat deposits. There it will form a calorific Trojan horse. If the subject attempts a little exercise, these deposits may be called upon to supply energy. But fat must be hydrolysed before it can be released. Once again the endless cycle of hydrolysis and recombination will be set up; once again energy will be lost from the body as heat. The subject will find his exercise warming but not particularly exhausting - exhaustion is a muscular effect. But it will slim him dramatically. Daedalus may have invented a diet and exercise regime that really works!

David Jones 